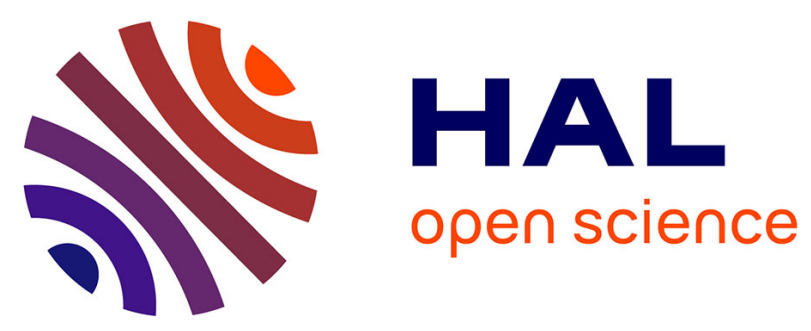

\title{
Out-of-plane precession of an in-plane magnetized free layer submitted to the spin-transfer torque of a perpendicular polarizer: An analytical perturbative approach
}

\author{
B. Lacoste, L. D. Buda-Prejbeanu, U. Ebels, B. Dieny
}

\section{To cite this version:}

B. Lacoste, L. D. Buda-Prejbeanu, U. Ebels, B. Dieny. Out-of-plane precession of an in-plane magnetized free layer submitted to the spin-transfer torque of a perpendicular polarizer: An analytical perturbative approach. Physical Review B: Condensed Matter and Materials Physics (1998-2015), 2013, 88, pp.054425. 10.1103/PhysRevB.88.054425 . cea-01072718

\section{HAL Id: cea-01072718 https://hal-cea.archives-ouvertes.fr/cea-01072718}

Submitted on 28 Apr 2021

HAL is a multi-disciplinary open access archive for the deposit and dissemination of scientific research documents, whether they are published or not. The documents may come from teaching and research institutions in France or abroad, or from public or private research centers.
L'archive ouverte pluridisciplinaire HAL, est destinée au dépôt et à la diffusion de documents scientifiques de niveau recherche, publiés ou non, émanant des établissements d'enseignement et de recherche français ou étrangers, des laboratoires publics ou privés. 


\title{
Out-of-plane precession of an in-plane magnetized free layer submitted to the spin-transfer torque of a perpendicular polarizer: An analytical perturbative approach
}

\author{
B. Lacoste, L. D. Buda-Prejbeanu, U. Ebels, and B. Dieny \\ SPINTEC, UMR CEA/CNRS/UJF-Grenoble 1/ Grenoble-INP, INAC, Grenoble, F-38054, France
}

(Received 4 June 2013; revised manuscript received 15 July 2013; published 28 August 2013)

\begin{abstract}
An analytical perturbative approach is presented to calculate the out-of-plane precessional motion of the magnetization of an in-plane free layer submitted to the spin torque of a perpendicular polarizer in the macrospin model. We consider the effects of the uniaxial anisotropy field, of an in-plane applied field, and of the perturbation due to the in-plane reference layer on the otherwise circular and monochromatic oscillations of the free layer. We calculate the frequency change due to these perturbations, the amplitude of the second harmonics, and the critical current for the existence of oscillations. This approach is rather general in the treatment of harmonics in spin torque oscillators.
\end{abstract}

DOI: 10.1103/PhysRevB.88.054425

PACS number(s): 72.25.Ba, 75.78.-n, 75.40.Gb, 85.70.Kh

\section{INTRODUCTION}

Spin torque oscillators (STOs) are spintronics nanodevices that produce radio frequencies in the gigahertz range with a dc current. ${ }^{1-3}$ Due to spin-transfer torque, a spin polarized current flowing through a free magnetized layer is able to modify its magnetization orientation, producing self-sustained oscillations of the free-layer magnetization ${ }^{4}$ that can be measured through a variation of the giant magnetoresistance (GMR), for spin valves, or the tunnel magnetoresistance (TMR) for magnetic tunnel junctions (MTJ). While most devices take advantage of in-plane precession (IPP) of the freelayer magnetization, ${ }^{5}$ recent studies have shown the possibility of fabricating STOs in which the free-layer magnetization undergoes out-of-plane precessional motion (OPP). ${ }^{6,7}$ Such devices are based on an in-plane magnetized MTJ, comprised of an in-plane magnetized free layer and an in-plane analyzer producing a magnetoresistive signal, with the addition of a perpendicular polarizer. The effect of the spin-transfer torque (STT) due to the perpendicular polarizer is to pull the free-layer magnetization out of plane, and due to the strong demagnetizing field, the free-layer magnetization then precesses around the direction normal to the plane. One advantage of the OPP over IPP is the large amplitude of the OPP oscillations, which gives a larger resistance variation, and therefore a larger RF output signal.

The most simple macrospin models of an in-plane MTJ with a perpendicular polarizer take into account only the demagnetizing field and the spin torque from the perpendicular polarizer, ${ }^{8,9}$ as more general models do not seem to be solvable analytically. Here, we present a perturbative approach that allows to estimate the effect of the applied field, the anisotropy field, and the spin torque from the analyzer on the OPP state. We were able to compute the change in frequency due to these perturbations and the critical current for the existence of the OPP steady state submitted to these perturbations.

This approach is different from earlier studies of the STO dynamics ${ }^{10-12}$ for which the dynamical steady state was calculated as a small deviation from the equilibrium state. It differs also from the planar approximation, ${ }^{13}$ which applies in the assumption that the dynamics is almost in-plane. Here, the dynamical steady state is a deviation from another limit cycle with a simpler expression.
Summary of results. Let $z_{0}<1$ be the average out-of-plane angle of the free-layer magnetization, which is proportional to the applied current $I: z_{0}=\frac{\hbar}{2 e} \frac{\eta_{\mathrm{PERP}} I}{\alpha \mu_{0} M_{s}^{2} V}$. The main results of this paper are summarized in the table below, where we present the frequency change from the unperturbed frequency $f_{0}$, the amplitude of the second harmonic, and the critical current $I_{c 4}$, the lower boundary for the existence of OPP, with respect to the perturbation due to an in-plane applied field, $H_{x}$ or $H_{y}$, due to the in-plane uniaxial anisotropy field $H_{k}$, and due to the STT of the reference layer $p_{x}$ :

\begin{tabular}{lccc}
\hline \hline & $H_{x}, H_{y}$ & $H_{k}$ & $p_{x}$ \\
\hline$\left(f-f_{0}\right) / f_{0}$ & $-\frac{1}{2 z_{0}^{4}}\left(\frac{H_{x / y}}{M_{S}}\right)^{2}$ & $-\frac{1}{32 z_{0}^{4}}\left(\frac{H_{k}}{M_{S}}\right)^{2}$ & $\frac{1}{2}\left(\frac{p_{x}}{M_{S}}\right)^{2}$ \\
2nd harmonic & $H_{x / y} / z_{0}^{2}$ & 0 & $p_{x} / z_{0}$ \\
$I_{c 4} \sim \ldots$ & $\alpha \sqrt{H_{x / y} M_{s}}$ & $\alpha \sqrt{H_{k} M_{s}}$ & 0 \\
\hline \hline
\end{tabular}

\section{MACROSPIN EQUATION AND SOLVABLE CASE}

\section{A. LLGS equation}

In the system described in Fig. 1(a), the free-layer magnetization $\boldsymbol{m}$ is the solution of the following Landau-LifschitzGilbert-Slonczewski (LLGS) equation in the macrospin approximation:

$$
\begin{aligned}
\frac{\mathrm{d} \boldsymbol{m}}{\mathrm{dt}}= & -\mu_{0} \gamma\left(\boldsymbol{m} \times \boldsymbol{H}_{\mathrm{eff}}\right)+\alpha\left(\boldsymbol{m} \times \frac{\mathrm{d} \boldsymbol{m}}{\mathrm{dt}}\right) \\
& -\mu_{0} \gamma\left[\boldsymbol{m} \times\left(\boldsymbol{m} \times \boldsymbol{M}_{\mathrm{pol}}\right)\right] .
\end{aligned}
$$

Here, $\gamma$ is the gyromagnetic ratio, $\mu_{0}$ is the vacuum permeability, and $\alpha$ is the Gilbert damping constant. $\frac{\mathrm{d} \boldsymbol{m}}{\mathrm{dt}}$ is the time derivative of the free-layer magnetization $\boldsymbol{m} . \boldsymbol{H}_{\mathrm{eff}}$ is the effective field that derives from the magnetic free energy $E_{0}$, and $\boldsymbol{M}_{\mathrm{pol}}$ is the spin torque vector:

$$
\begin{aligned}
\boldsymbol{H}_{\mathrm{eff}} & =-\frac{1}{\mu_{0} M_{S} V} \frac{\partial E_{0}}{\partial \boldsymbol{m}}, \\
\boldsymbol{M}_{\mathrm{pol}} & =-a_{J \text { LONG }} \boldsymbol{m}_{\mathrm{LONG}}+a_{J} \text { PERP } \boldsymbol{m}_{\mathrm{PERP}} .
\end{aligned}
$$

$M_{S}$ is the saturation magnetization and $V$ is the volume of the layer. $\boldsymbol{m}_{\mathrm{LONG}}$ (respectively, $\boldsymbol{m}_{\mathrm{PERP}}$ ) is the unit vector 
(a)

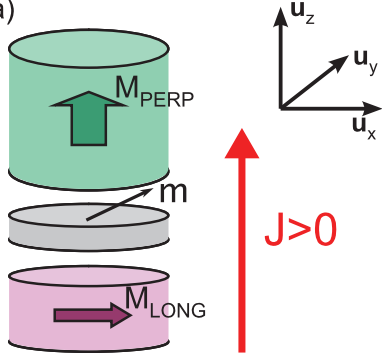

(b)

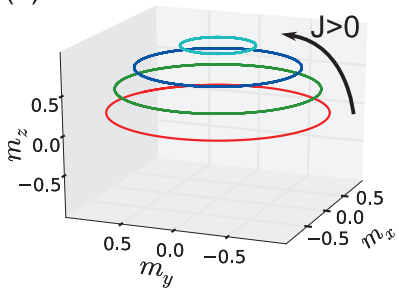

(c)

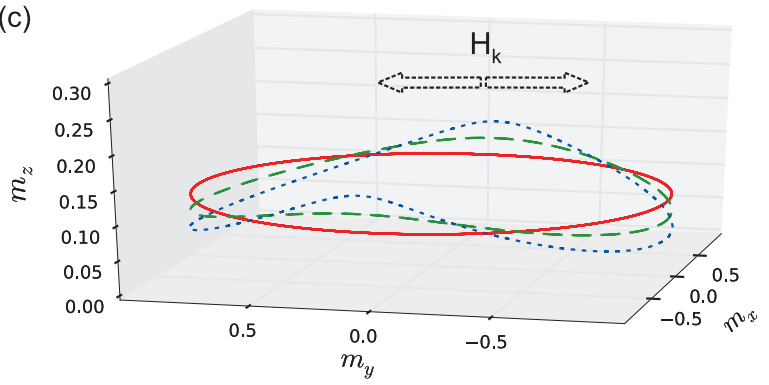

FIG. 1. (Color online) (a) Schematics of the in-plane MTJ with a perpendicular polarizer. The applied field can be in any direction. (b) Unperturbed trajectory for different current density $J: 2,6,10$, and $14 \times 10^{11} \mathrm{~A} / \mathrm{m}^{2}$. (c) Perturbed trajectory for $J=1.5 \times 10^{11} \mathrm{~A} / \mathrm{m}^{2}$ and different anisotropy field $H_{k}: 0,24$, and $48 \mathrm{kA} / \mathrm{m}$.

collinear to the magnetization of the longitudinal (respectively, perpendicular) polarizer. Namely, $\boldsymbol{m}_{\mathrm{LONG}}=\boldsymbol{u}_{x}$ and $\boldsymbol{m}_{\mathrm{PERP}}=$ $\boldsymbol{u}_{z}$. There is a minus sign in front of the term coming from the longitudinal polarizer because the perpendicular polarizer is situated above the free layer, whereas the perpendicular polarizer is below. For $i=(\mathrm{LONG}, \mathrm{PERP}), a_{J i}$ is the spin torque amplitude due to the corresponding polarizing layer. Its expression is given by ${ }^{14}$

$$
a_{J i}=\frac{\hbar}{2 e} \frac{J}{\mu_{0} M_{S} t} \frac{\eta_{i}}{1+\lambda_{i} \boldsymbol{m} \cdot \boldsymbol{m}_{i}},
$$

where $t$ is the free-layer thickness, $\eta_{\text {LONG }}$ and $\eta_{\text {PERP }}$ are the spin polarizations of the polarizing layers, and we call $\lambda_{\text {LONG }}$ and $\lambda_{\text {PERP }}$ the spin polarization asymmetries. The spin polarization asymmetry depends on the nature of the nonmagnetic spacer layer separating the free layer from the polarizer, it is almost zero for a tunnel barrier and around 0.5 for a copper spacer. ${ }^{14,15}$

Equation (1) can be written in a more convenient way, with $\gamma_{0}=\mu_{0} \gamma /\left(1+\alpha^{2}\right)$, as

$$
\begin{aligned}
\frac{\mathrm{d} \boldsymbol{m}}{\mathrm{dt}}= & -\gamma_{0} \boldsymbol{m} \times \boldsymbol{H}_{\mathrm{eff}}-\gamma_{0} \alpha \boldsymbol{m} \times\left(\boldsymbol{m} \times \boldsymbol{H}_{\mathrm{eff}}\right) \\
& +\gamma_{0} \alpha \boldsymbol{m} \times \boldsymbol{M}_{\mathrm{pol}}-\gamma_{0} \boldsymbol{m} \times\left(\boldsymbol{m} \times \boldsymbol{M}_{\mathrm{pol}}\right) .
\end{aligned}
$$

For convenience, we introduce $P_{0}$, which we call the spin torque potential, given by

$$
\begin{aligned}
P_{0}=- & \frac{\hbar}{2 e} J S \eta_{\mathrm{LONG}} \frac{\ln \left(1+\lambda_{\mathrm{LONG}} \boldsymbol{m}_{\mathrm{LONG}} \cdot \boldsymbol{m}\right)}{\lambda_{\mathrm{LONG}}} \\
& +\frac{\hbar}{2 e} J S \eta_{\mathrm{PERP}} \frac{\ln \left(1+\lambda_{\mathrm{PERP}} \boldsymbol{m}_{\mathrm{PERP}} \cdot \boldsymbol{m}\right)}{\lambda_{\mathrm{PERP}}} .
\end{aligned}
$$

Here, $S$ is the free-layer area through which the current flows. The spin torque vector $\boldsymbol{M}_{\text {pol }}$ derives from $P_{0}$ :

$$
\boldsymbol{M}_{\mathrm{pol}}=\frac{1}{\mu_{0} M_{S} V} \frac{\partial P_{0}}{\partial \boldsymbol{m}} .
$$

$P_{0}$ has the advantage of being coordinate independent, making the change of variables easier. For $\lambda_{\mathrm{LONG}}=0, P_{0}$ is defined by taking the limit when $\lambda_{\text {LONG }} \rightarrow 0$ and similarly for $\lambda_{\text {PERP }}=0$.

We also introduce the two scalar functions $\mathcal{H}$ and $\mathcal{G}$, which are base invariant:

$$
\mathcal{H}=\gamma_{0} \frac{E_{0}+\alpha P_{0}}{\mu_{0} M_{S} V}, \quad \mathcal{G}=\gamma_{0} \frac{\alpha E_{0}-P_{0}}{\mu_{0} M_{S} V} .
$$

Equation (2) becomes

$$
\frac{\mathrm{d} \boldsymbol{m}}{\mathrm{dt}}=\boldsymbol{m} \times \nabla_{\boldsymbol{m}} \mathcal{H}+\boldsymbol{m} \times\left(\boldsymbol{m} \times \nabla_{\boldsymbol{m}} \mathcal{G}\right)
$$

$\mathcal{H}$ is equivalent to a Hamiltonian, it is a conservative term, whereas $\mathcal{G}$ is a dissipative term. Without spin torque, $\mathcal{H}$ and $\mathcal{G}$ are proportional, their ratio being $\alpha$, but this simple relation is not valid anymore when we take into account the spin-transfer torque.

Using the condensed notation $F_{X}=\frac{\partial F}{\partial X}$, Eq. (4) simplifies in spherical coordinates:

$$
\begin{gathered}
m_{x}=\sin \theta \cos \phi, \\
m_{y}=\sin \theta \sin \phi, \\
m_{z}=\cos \theta \\
\dot{\theta}=-\frac{\mathcal{H}_{\phi}}{\sin \theta}-\mathcal{G}_{\theta}, \quad \dot{\phi} \sin \theta=\mathcal{H}_{\theta}-\frac{\mathcal{G}_{\phi}}{\sin \theta} .
\end{gathered}
$$

In the following, we consider a thin free layer. Its magnetic free energy is the sum of three contributions, respectively, the demagnetizing energy, a uniaxial anisotropy energy, and the Zeeman energy:

$$
E_{0}=\frac{1}{2} \mu_{0} V M_{S}^{2} m_{z}^{2}+K_{u} V\left(1-m_{x}^{2}\right)-\mu_{0} M_{S} V \boldsymbol{m} \cdot \boldsymbol{H}_{a} .
$$

$K_{u}$ is a uniaxial anisotropy energy constant, related to the uniaxial anisotropy effective field $H_{k}=\frac{2 K_{u}}{\mu_{0} M_{S}}$. The uniaxial anisotropy accounts for magnetocrystalline and shape anisotropy and it favors $x$-axis orientation, which is the easy axis of magnetization. Here, the applied field $\boldsymbol{H}_{a}=$ $\left(H_{x}, H_{y}, H_{z}\right)$ is in an arbitrary direction, with norm $H_{a}$.

The two scalar functions defined above, the conservative $\mathcal{H}$ and dissipative $\mathcal{G}$ terms, have the following expressions in spherical coordinates:

$$
\begin{aligned}
\mathcal{H}= & \gamma_{0}\left[\frac{M_{S}}{2} \cos ^{2} \theta-\frac{H_{k}}{2} \sin ^{2} \theta \cos ^{2} \phi-H_{x} \sin \theta \cos \phi\right. \\
& -H_{y} \sin \theta \sin \phi-H_{z} \cos \theta \\
& \left.+\frac{\alpha p_{z}}{\lambda_{z}} \ln \left(1+\lambda_{z} \cos \theta\right)-\frac{\alpha p_{x}}{\lambda_{x}} \ln \left(1+\lambda_{x} \sin \theta \cos \phi\right)\right],
\end{aligned}
$$




$$
\begin{aligned}
\mathcal{G}= & \gamma_{0}\left[\frac{p_{x}}{\lambda_{x}} \ln \left(1+\lambda_{x} \sin \theta \cos \phi\right)-\frac{p_{z}}{\lambda_{z}} \ln \left(1+\lambda_{z} \cos \theta\right)\right. \\
& +\alpha \frac{M_{S}}{2} \cos ^{2} \theta-\alpha \frac{H_{k}}{2} \sin ^{2} \theta \cos ^{2} \phi \\
& \left.-\alpha H_{x} \sin \theta \cos \phi-\alpha H_{y} \sin \theta \sin \phi-\alpha H_{z} \cos \theta\right] .
\end{aligned}
$$

Here, $p_{x}=\frac{\hbar}{2 e} \frac{J \eta_{\mathrm{LONG}}}{\mu_{0} M_{S} t}, p_{z}=\frac{\hbar}{2 e} \frac{J \eta_{\mathrm{PERP}}}{\mu_{0} M_{S} t}, \lambda_{x}=\lambda_{\mathrm{LONG}}$, and $\lambda_{z}=$ $\lambda_{\text {PERP }}$ for compactness. The demagnetizing energy is assumed, henceforth, to be the dominant term of the magnetic free energy. In fact, we suppose that $M_{S} \gg H_{k}, H_{a}, a_{J}$, which is generally the case for thin films in nanopillars. Therefore the dominant term of the conservative part $\mathcal{H}$ comes from the demagnetizing energy $\frac{M_{S}}{2} \cos ^{2} \theta$. The dissipative term $\mathcal{G}$ is smaller compared to the conservative part, by a factor $\alpha$, and it is dominated by the spin torque $p_{z} \cos \theta /\left(1+\lambda_{z} \cos \theta\right)$ and the damping of the demagnetizing field $\alpha \frac{M_{S}}{2} \cos ^{2} \theta$. The uniaxial anisotropy field and the Zeeman field give a smaller contribution. As shown in Fig. 1(c), changing the anisotropy field, for instance, does not change much the magnetization trajectory. In fact, the trajectory of the dynamical system with a uniaxial anisotropy, an applied field, or an analyzer spin torque is very close to the trajectory with none of them. Hence it is reasonable to study first the simplest dynamical system describing a limit cycle, and then to treat the addition of other terms as perturbations around this limit cycle.

\section{B. Unperturbed system}

In a first attempt to describe the out-of-plane precession state of the free-layer magnetization with a perpendicular polarizer, we will consider only the demagnetization energy $\frac{M_{S}}{2} \cos ^{2} \theta$ in the total energy, and only the $z$ component of the spin torque $p_{z}$, in the particular case of an MTJ $\left(\lambda_{z}=0\right.$ and $\left.P_{0}=p_{z} m_{z}\right){ }^{8,16}$ Neglecting other terms, the trajectory is exactly circular [see Fig. 1(c)], and because they are the dominant terms, it is a good approximation of the complicated trajectory including all the terms. In this framework, the Hamiltonian and dissipative terms have simple expressions:

$$
\begin{aligned}
\mathcal{H} & =\frac{\gamma_{0} M_{S}}{2} \cos ^{2} \theta+\alpha \gamma_{0} p_{z} \cos \theta, \\
\mathcal{G} & =\alpha \frac{\gamma_{0} M_{S}}{2} \cos ^{2} \theta-\gamma_{0} p_{z} \cos \theta,
\end{aligned}
$$

The equation of motion then writes as:

$$
\begin{aligned}
\dot{\theta} & =\alpha \gamma_{0} M_{S} \cos \theta \sin \theta-\gamma_{0} p_{z} \sin \theta, \\
\dot{\phi} \sin \theta & =-\gamma_{0} M_{S} \cos \theta \sin \theta-\alpha \gamma_{0} p_{z} \sin \theta .
\end{aligned}
$$

We set: $\quad \omega_{0}=\frac{\gamma_{0} p_{z}}{\alpha}$ and $\quad z_{0}=\frac{p_{z}}{\alpha M_{S}}$.

With the change of variables $z=\cos \theta$, Eq. (6) becomes

$$
\begin{aligned}
& \dot{z}=-\alpha \omega_{0}\left(1-z^{2}\right)\left(\frac{z}{z_{0}}-1\right), \\
& \dot{\phi}=-\omega_{0}\left(\frac{z}{z_{0}}+\alpha^{2}\right) .
\end{aligned}
$$

This equation can be solved explicitly for the variable $z$ by separation of variables and using partial fraction decomposition:

$$
\left|\frac{z-z_{0}}{z_{i}-z_{0}}\right|\left|\frac{(1+z)\left(1-z_{i}\right)}{(1-z)\left(1+z_{i}\right)}\right|^{z_{0} / 2} \sqrt{\frac{1-z_{i}^{2}}{1-z^{2}}}=e^{-\beta_{0} \omega_{0} t} .
$$

Here, $z_{i}$ and $\phi_{i}$ are the initial out-of-plane magnetization and in-plane phase. The initial time is taken to be zero. We set $\beta_{0}=\alpha\left(1-z_{0}^{2}\right) / z_{0}$, with the inverse relaxation time $\beta_{0} \omega_{0}=$ $\alpha M_{S} \gamma_{0}\left(1-z_{0}^{2}\right)$ always positive. As the left-hand side tends towards zero when time tends to infinity, the right-hand side must also tend towards zero. Except for the degenerate case of $z_{i}= \pm 1$, this condition means that $z \rightarrow z_{0}$ for long-term time. Therefore after a certain time, the condition $\left|z-z_{0}\right| \ll 1$ will be fulfilled, and we can derive an approximation for the out-of-plane magnetization $z$ and the phase $\phi$ :

$$
\begin{aligned}
& z-z_{0}=\left(z_{i}-z_{0}\right) e^{-\beta_{0} \omega_{0} t}, \\
& \phi-\phi_{i}=-\omega_{0}\left(1+\alpha^{2}\right) t+\frac{z_{i}-z_{0}}{z_{0} \beta_{0}}\left(e^{-\beta_{0} \omega_{0} t}-1\right) .
\end{aligned}
$$

Equation (8) describes the unperturbed out-of-plane precession of the magnetization. In permanent regime, the precession is characterized by a constant out-of-plane magnetization $m_{z}=z_{0}$ and a constant frequency $f_{0}$, which is proportional to the applied current $J^{9}$ [see Fig. 1(b)]:

$$
f_{0}=\frac{\omega_{0}\left(1+\alpha^{2}\right)}{2 \pi}=\frac{\gamma \hbar \eta_{\mathrm{PERP}}}{4 \pi e \alpha M_{S} t} J .
$$

This trajectory is in fact an attractive limit-cycle of the dynamical system described by Eq. (7). In the general case, the study of limit-cycle stability requires to define a Poincaré map but in the present case, where the geometrical space is two dimensional, one can directly extract the eigenvalue of the linearized Poincaré map, also known as multiplier $\mu_{1}$ :

$$
\mu_{1}=\exp \left\{\int_{0}^{T} \operatorname{div} \boldsymbol{F}\left[\boldsymbol{x}_{0}(t)\right] d t\right\}
$$

where $T$ is the period of the limit cycle, $\boldsymbol{F}(x)$ is the vector field of the dynamical system $\dot{\boldsymbol{x}}=\boldsymbol{F}(\boldsymbol{x})$, and $\boldsymbol{x}_{0}(t)$ is a parameterization of the limit cycle. Expressed in spherical coordinates, Eq. (10) can be simplified with the expression of the vector field taken from the left-hand side of Eq. (5):

$$
\begin{aligned}
\mu_{1}= & \exp \left\{-\int_{0}^{T}\left[\cot \theta \mathcal{G}_{\theta}\left(\boldsymbol{x}_{0}(t)\right)\right.\right. \\
& \left.\left.+\mathcal{G}_{\theta \theta}\left(\boldsymbol{x}_{0}(t)\right)+\frac{\mathcal{G}_{\phi \phi}\left(\boldsymbol{x}_{0}(t)\right)}{\sin ^{2} \theta}\right] d t\right\} .
\end{aligned}
$$

Notice that only the dissipative term appears in this expression. In the simplified case of Eq. (7), the timeparameterized spherical equation of the limit cycle is $\boldsymbol{x}_{0}(t)=$ $\left[\theta_{0},-\omega_{0}\left(1+\alpha^{2}\right) t\right]\left(z_{0}=\cos \theta_{0}\right)$. The expression of $\operatorname{div} \boldsymbol{F}$ is then $-\gamma_{0} p_{z} \frac{1+2 z_{0} \cos \theta-3 \cos ^{2} \theta}{z_{0}}$ and the period $T=1 / f_{0}=$ $\frac{2 \pi}{\omega_{0}\left(1+\alpha^{2}\right)}$. The multiplier of the Poincaré map is then $\mu_{1}=$ $\exp \left(-\frac{2 \pi \beta_{0}}{1+\alpha^{2}}\right)$. The multiplier is the relaxation rate towards the limit cycle from a state slightly deviated, for instance, by thermal fluctuations. ${ }^{16}$ Because $\mu_{1}$ is less than unity in absolute value, the limit cycle is hyperbolically stable. 


\section{High-symmetry system}

In addition to the previous simple case, we can add some terms that present the same symmetry as the unperturbed equation, as treated in previous papers. ${ }^{8,16}$ We consider terms that are independent of the in-plane angle $\phi$ : the spin torque amplitude is angle dependent $\left(\lambda_{z} \neq 0\right)$ and there is an out-ofplane applied field $H_{z}$. The out-of-plane applied can describe the stray field created by the perpendicular polarizer, or an external applied field. Eq. (5) is then

$$
\begin{aligned}
\dot{\theta} & =\alpha \gamma_{0} M_{S} \cos \theta \sin \theta-\frac{\gamma_{0} p_{z} \sin \theta}{1+\lambda_{z} \cos \theta}-\alpha \gamma_{0} H_{z} \sin \theta, \\
\dot{\phi} \sin \theta & =-\gamma_{0} M_{S} \cos \theta \sin \theta-\frac{\alpha \gamma_{0} p_{z} \sin \theta}{1+\lambda_{z} \cos \theta}+\gamma_{0} H_{z} \sin \theta .
\end{aligned}
$$

As for the unperturbed system, the vector field is only $\theta$ dependent, hence we can solve analytically the system. We expect to find at least one limit cycle, so we directly look for the permanent regime solution, with a constant angle $\theta$. By changing the variables $z=\cos \theta$ and using the parameters $\omega_{0}$, $z_{0}$, and $v_{z}=\gamma_{0} H_{z} / \omega_{0}$, one can directly find the (nontrivial) solutions $\left(z_{+}, z_{-}\right)$of $\dot{z}=0$ :

$$
z_{ \pm}=-\frac{1-\lambda_{z} z_{0} \nu_{z}}{2 \lambda_{z}} \pm \frac{1}{2 \lambda_{z}} \sqrt{\left(1+\lambda_{z} z_{0} \nu_{z}\right)^{2}+4 \lambda_{z} z_{0}}
$$

The positive solution $z_{+}$tends toward $z_{0}$ when the parameters $\lambda$ and $\nu_{z}$ tend toward zero: it is the perturbed solution. As we are interested here in solutions with a small out-of-plane magnetization $z$ for application in precessional switching, we will focus only on the $z_{+}$solution. Notice that $z_{-}$does not always exist (if $\left|z_{-}\right|>1$ for instance), and when it does, it does not always describe an attractive limit cycle. With the constant out-of-plane magnetization $z_{+}$, the frequency of the precession is also changed compared to the unperturbed frequency $f_{0}=$ $\omega_{0}\left(1+\alpha^{2}\right) / 2 \pi$ :

$$
f=\frac{f_{0}}{1+\lambda_{z} z_{+}} .
$$

For a small $\lambda_{z}$ parameter, we obtain the approximation by Taylor expansion $f=f_{0}\left[1-\lambda_{z} z_{0}\left(1+v_{z}\right)\right]$. See Fig. 2 for comparison of the analytical expression of the frequency with $\lambda \neq 0$ and numerical simulations.

The high-symmetry system is not of real interest for applications in precessional switching, as an important parameter is omitted, the uniaxial anisotropy. However, since the approximation at order 1 is close to the exact solution, this gives credit to this approach and encourages the investigation of other additional perturbative terms.

The stability of the out-of-plane precession state (OPP) is insured by the condition $\mu_{1}<1$. It also means that if LLGS (6) is slightly modified, for instance, by adding the term associated with a uniaxial anisotropy, the OPP state will remain a limit cycle, for small enough perturbations. ${ }^{16}$ In the following section, we will start from Eq. (6), henceforth called the unperturbed case, and study the general case with the general assumption that the solutions are very close to the unperturbed case. The unperturbed dynamical system will be modified by perturbations, namely, uniaxial anisotropy field, applied field, and longitudinal polarizer.

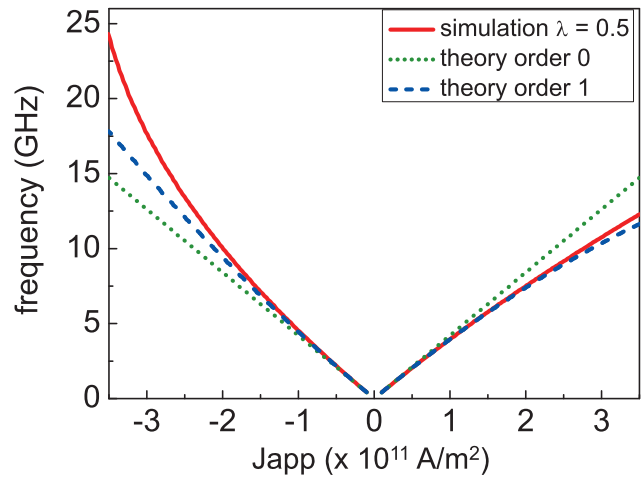

FIG. 2. (Color online) Oscillation frequency vs applied current density $J$. Comparison between macrospin simulation with $\lambda_{z}=0.5$ (red full line) and analytical expressions at order 0 in $\lambda_{z}$ (green dotted line) and order 1 (blue dashed line). The other simulation parameters are $\alpha=0.02, M_{S}=1.2 \times 10^{6} \mathrm{~A} / \mathrm{m}, \eta_{\mathrm{PERP}}=0.6, t=$ $3 \mathrm{~nm}$, and $H_{z}=0$.

\section{PERTURBATION THEORY}

\section{A. Uniaxial anisotropy}

Compared to the previous case, including the uniaxial anisotropy term in Eq. (5) makes the vector field not only $\theta$-dependent but also $\phi$-dependent. Exact analytical solutions are then much more difficult to obtain, so we use a perturbative approach to approximate the solution, ${ }^{17}$ using a Lindstedt's expansion. ${ }^{18}$ When a periodic dynamical system is perturbed, the perturbated solution will also be periodic, by continuity. The perturbated solution is approximated by a generalized asymptotic expansion that preserves the periodicity of the solution, the Lindstedt's series for which the frequency is also expanded with respect to the perturbation parameter $\epsilon$. The only assumption on the perturbation parameter is that it is small compared to the nonperturbated frequency, hence the equations are normalized to the frequency. Thus the approximation error is of order $\mathrm{O}(\epsilon)$ on a time scale of $\mathrm{O}(1 / \epsilon)$, which is sufficient if the functions are periodic with a normalized period of 1 .

On top of the uniaxial anisotropy perturbation, all the following cases will also be solved using this approach. From the unperturbed Eq. (6), we add the uniaxial anisotropy term:

$$
\begin{aligned}
\dot{\theta}= & \gamma_{0}\left[\alpha M_{S} \cos \theta \sin \theta-p_{z} \sin \theta-\frac{H_{k}}{2} \sin \theta \sin (2 \phi)\right. \\
& \left.+\alpha \frac{H_{k}}{2} \sin \theta \cos \theta(1+\cos (2 \phi))\right], \\
\dot{\phi} \sin \theta= & \gamma_{0}\left[-M_{S} \cos \theta \sin \theta-\alpha \frac{H_{k}}{2} \sin \theta \sin (2 \phi)\right. \\
& \left.-\alpha p_{z} \sin \theta-\frac{H_{k}}{2} \sin \theta \cos \theta(1+\cos (2 \phi))\right] .
\end{aligned}
$$

We use again the notation $\omega_{0}, z_{0}$, and $\epsilon$ defined below. For clarity, the time variable is changed to $\tau$ :

$$
\epsilon=\frac{\alpha}{1+\alpha^{2}} \frac{H_{k}}{2 p_{z}}, \quad \tau=\omega_{0}\left(1+\alpha^{2}\right) t .
$$


The time derivative $\dot{X}$ is also changed to $X^{\prime}$, which means a derivative with respect to $\tau$. We define $r_{0}=\sqrt{1-z_{0}^{2}}$ and $\beta=\alpha r_{0}^{2} /\left[z_{0}\left(1+\alpha^{2}\right)\right]$. As $\alpha \ll 1$, the parameter $\beta$ is very close to $\beta_{0}$ previously defined. For convenience, we use the functions

$$
\begin{aligned}
& g_{\theta}(\theta)=\frac{\alpha}{1+\alpha^{2}} \sin \theta\left(\frac{\cos \theta}{z_{0}}-1\right), \\
& h_{\phi}(\theta)=-\frac{\cos \theta+z_{0} \alpha^{2}}{z_{0}\left(1+\alpha^{2}\right)} \sin \theta .
\end{aligned}
$$

We also define two perturbation functions, which in the case of the uniaxial anisotropy have the following form:

$$
\begin{aligned}
& f_{\theta}^{\epsilon}(\theta, \phi)=-\sin \theta \sin (2 \phi)+\alpha \sin \theta \cos \theta(1+\cos (2 \phi)), \\
& f_{\phi}^{\epsilon}(\theta, \phi)=-\sin \theta \cos \theta(1+\cos (2 \phi))-\alpha \sin \theta \sin (2 \phi) .
\end{aligned}
$$

Hence Eq. (13) takes the simplified form

$$
\begin{aligned}
\theta^{\prime} & =g_{\theta}(\theta)+\epsilon f_{\theta}^{\epsilon}(\theta, \phi), \\
\phi^{\prime} \sin \theta & =h_{\phi}(\theta)+\epsilon f_{\phi}^{\epsilon}(\theta, \phi) .
\end{aligned}
$$

To solve this system, the following assumptions are made: (i) $\epsilon \ll 1$ (see Appendix) and (ii) the functions $\theta(\tau)$ and $\phi(\tau)$, solutions of Eq. (16), are written as a power series of the parameter $\epsilon$. In the framework of the Lindstedt's series, we also consider that the period of the limit cycle can be written as a power series of $\epsilon$, to get rid of the secular terms. In fact, we change the time variable $\tau^{\prime}=\omega \tau$. Hence $\omega, \theta$, and $\phi$ will be written (up to the second order) as

$$
\begin{aligned}
\theta & =\theta_{0}+\epsilon \theta_{1}+\epsilon^{2} \theta_{2}+\ldots, \\
\phi & =\phi_{0}+\epsilon \phi_{1}+\epsilon^{2} \phi_{2}+\ldots, \\
\omega & =1+\epsilon f_{1}+\epsilon^{2} f_{2}+\ldots
\end{aligned}
$$

The development of the calculations is made in Appendix. For the uniaxial anisotropy, we find the trajectory equations up to first order:

$$
\begin{gathered}
\theta_{0}\left(\tau^{\prime}\right)=\arccos \left(z_{0}\right), \quad \phi_{0}\left(\tau^{\prime}\right)=-\tau^{\prime}, \\
\left\{\begin{array}{l}
\theta_{1}\left(\tau^{\prime}\right)=a_{0}+a_{2} \cos \left(2 \tau^{\prime}\right)+b_{2} \sin \left(2 \tau^{\prime}\right), \\
\phi_{1}\left(\tau^{\prime}\right)=c_{2} \cos \left(2 \tau^{\prime}\right)+d_{2} \sin \left(2 \tau^{\prime}\right),
\end{array}\right.
\end{gathered}
$$

with the coefficients defined by:

$$
\begin{aligned}
& a_{0}=\left(1+\alpha^{2}\right) z_{0}^{2} / r_{0}, \\
& a_{2}=\frac{r_{0}}{4+\beta^{2}}\left(\alpha \beta z_{0}-2\right), \\
& b_{2}=\frac{r_{0}}{4+\beta^{2}}\left(2 \alpha z_{0}+\beta\right), \\
& c_{2}=-\frac{\left(1+\alpha^{2}\right) \beta^{2} / 2+\alpha \beta z_{0}+2 \alpha^{2}}{\alpha\left(4+\beta^{2}\right)}, \\
& d_{2}=-\frac{2 \alpha z_{0}+\beta}{\alpha\left(4+\beta^{2}\right)} .
\end{aligned}
$$

The frequency $f$ of the periodic orbit is changed only at the second order in the parameter $\epsilon$ :

$$
f_{1}=0, \quad f_{2} \approx-\frac{1+4 z_{0}^{2}}{8 z_{0}^{2}+2 \alpha^{2}} .
$$
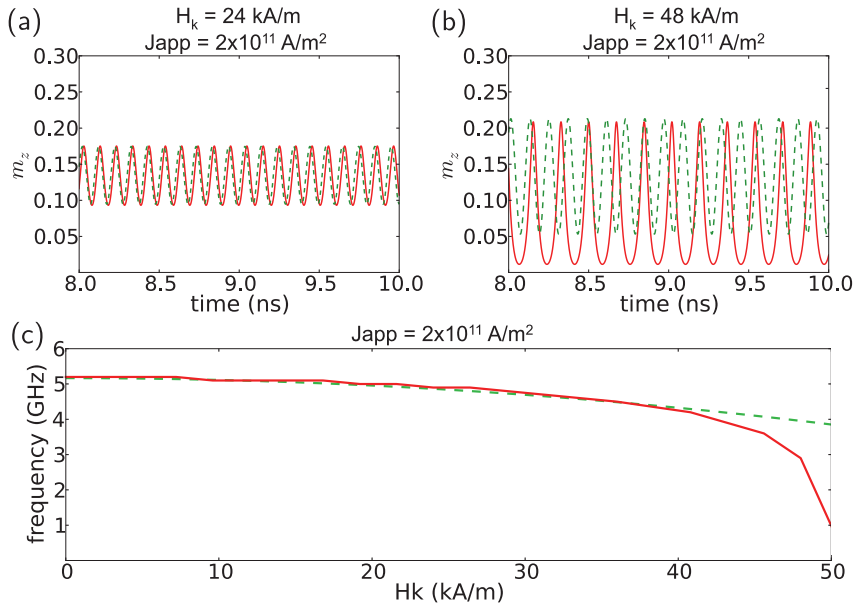

FIG. 3. (Color online) Macrospin simulation (red full line) and analytical expression at first order in $\epsilon$ (green dashed line). (a) and (b) Out-of plane component of the magnetization $m_{z}$ vs time, with applied current density of $J_{\text {app }}=2 \times 10^{11} \mathrm{~A} / \mathrm{m}^{2}\left(z_{0}=0.13\right)$ and with (a) $H_{k}=24 \mathrm{kA} / \mathrm{m}(\epsilon=0.05)$ and (b) $H_{k}=48 \mathrm{kA} / \mathrm{m}(\epsilon=0.1)$. (c) Frequency of the in-plane component $m_{x}$ vs anisotropy field $H_{k}$. The other used parameters are given in the text.

With the expression of the unperturbed frequency $f_{0}$ found in Eq. (9), we obtain

$$
f=f_{0}\left(1-\epsilon^{2} \frac{1+4 z_{0}^{2}}{8 z_{0}^{2}+2 \alpha^{2}}\right) .
$$

These analytical expressions were compared to the results of 10-ns-long macrospin simulations. For the simulations, $\alpha=$ $0.02, M_{S}=1.2 \times 10^{6} \mathrm{~A} / \mathrm{m}, t=3 \mathrm{~nm}$, and $\eta_{\text {PERP }}=0.2$.

Figures 3(a) and 3(b) show the out-of-plane component of the free-layer magnetization $m_{z}$ in the permanent regime ( $8 \mathrm{~ns}$ after the application of the current pulse) for an applied current density of $J_{\text {app }}=2 \times 10^{11} \mathrm{~A} / \mathrm{m}^{2}$. We compare the macrospin simulation in red full line with the analytical expression at first order in $\epsilon$, in green dashed line. In Fig. 3(a), for $H_{k}=$ $24 \mathrm{kA} / \mathrm{m}$, corresponding to $\epsilon=0.05$, the analytical expression is very similar to the macrospin simulations. The phase shift with the simulation is due to the transient regime that is not taken into account in the analytical expression. However, in Fig. 3(b), for $H_{k}=48 \mathrm{kA} / \mathrm{m}$, and so $\epsilon=0.1$, the trajectory from the macrospin simulation is not exactly sinusoidal and it cannot be described by the first-order approximation only. As seen also in Fig. 3(c), for larger value of the perturbation $H_{k}$, the frequency change is not well described by the second-order approximation anymore. Notice that the anisotropy induces a perturbation of twice the natural frequency because of the symmetry of the anisotropy term. The applied field and analyzer spin torque induce a perturbation at the natural frequency.

The trajectory of the magnetization is very close to the trajectory of the unperturbed system, as shown in Fig. 1(c). It validates the use of a perturbation expansion. The perturbed trajectory has a larger out-of-plane component $m_{z}$ close to the easy-axis $\pm \boldsymbol{u}_{x}$ directions, and $m_{z}$ is smaller close to the hard-axis directions $\pm \boldsymbol{u}_{y}$. The magnetization also spends more time along the $\boldsymbol{u}_{x}$ axis than along the $\boldsymbol{u}_{y}$ axis because the in-plane angle time derivative is smaller along the easy axis. 
The magnetoresistive signal that can be measured with an in-plane analyzer depends on $m_{x}=\sin \theta \cos \phi$. Therefore the signal has a fundamental frequency and higher-order harmonics, of which the amplitude can be estimated as

$$
\begin{aligned}
m_{x} & =\sin \left(\theta_{0}+\epsilon \theta_{1}\right) \cos \left(-\tau+\epsilon \phi_{1}\right) \\
& \approx r_{0} \cos \tau+\epsilon\left(z_{0} \theta_{1} \cos \tau+r_{0} \phi_{1} \sin \tau\right) .
\end{aligned}
$$

Because $\theta_{1}$ and $\phi_{1}$ oscillate at twice the fundamental frequency, the second harmonic vanishes.

\section{B. Applied field}

We next discuss the perturbative effect of the applied field along the easy axis $H_{x}$ and along the in-plane hard axis $H_{y}$. They are treated in the same section because they yield similar results. However, the respective perturbative developments were done separately.

We introduce the dimensionless variables

$$
v_{x}=\frac{\alpha}{1+\alpha^{2}} \frac{H_{x}}{p_{z}}, \quad v_{y}=\frac{\alpha}{1+\alpha^{2}} \frac{H_{y}}{p_{z}} .
$$

The perturbation functions on the angles $\theta$ and $\phi$ are, respectively,

$$
\begin{aligned}
& f_{\theta}^{\nu_{x}}(\theta, \phi)=\alpha \cos \theta \cos \phi-\sin \phi, \\
& f_{\phi}^{\nu_{x}}(\theta, \phi)=-\alpha \sin \phi-\cos \theta \cos \phi, \\
& f_{\theta}^{v_{y}}(\theta, \phi)=\alpha \cos \theta \sin \phi+\cos \phi, \\
& f_{\phi}^{\nu_{y}}(\theta, \phi)=\alpha \cos \phi-\cos \theta \sin \phi .
\end{aligned}
$$

After solving the system up to second order, we find the expressions of the perturbed frequencies and the perturbed trajectories for the two directions of the applied field. In both cases,

$$
\begin{gathered}
f_{1}=0, \quad f_{2}=-\frac{\left(1+\alpha^{2}\right)^{2}}{2 z_{0}^{2}+2 \alpha^{2}}, \\
\left\{\begin{array}{l}
\theta_{1}^{i}\left(\tau^{\prime}\right)=a_{1}^{i} \cos \tau^{\prime}+b_{1}^{i} \sin \tau^{\prime}, \\
\phi_{1}^{i}\left(\tau^{\prime}\right)=c_{1}^{i} \cos \tau^{\prime}+d_{1}^{i} \sin \tau^{\prime},
\end{array}\right.
\end{gathered}
$$

with the coefficients for the field along the $\mathrm{x}$ axis given by:

$$
\begin{aligned}
a_{1}^{x} & =-\frac{1-\alpha \beta z_{0}}{1+\beta^{2}}, \\
b_{1}^{x} & =\frac{\beta+\alpha z_{0}}{1+\beta^{2}}, \\
c_{1}^{x} & =-\frac{\alpha \beta z_{0}+\beta^{2}\left(1+\alpha^{2}\right)+\alpha^{2}}{\alpha r_{0}\left(1+\beta^{2}\right)}, \\
d_{1}^{x} & =-\frac{\beta+\alpha z_{0}}{\alpha r_{0}\left(1+\beta^{2}\right)},
\end{aligned}
$$

and the coefficients for the field along the $\mathrm{y}$ axis given by:

$$
\begin{aligned}
a_{1}^{y} & =\frac{\beta+\alpha z_{0}}{1+\beta^{2}}, \\
b_{1}^{y} & =\frac{1-\alpha \beta z_{0}}{1+\beta^{2}}, \\
c_{1}^{y} & =-\frac{\beta+\alpha z_{0}}{\alpha r_{0}\left(1+\beta^{2}\right)}, \\
d_{1}^{y} & =\frac{\alpha \beta z_{0}+\beta^{2}\left(1+\alpha^{2}\right)+\alpha^{2}}{\alpha r_{0}\left(1+\beta^{2}\right)} .
\end{aligned}
$$
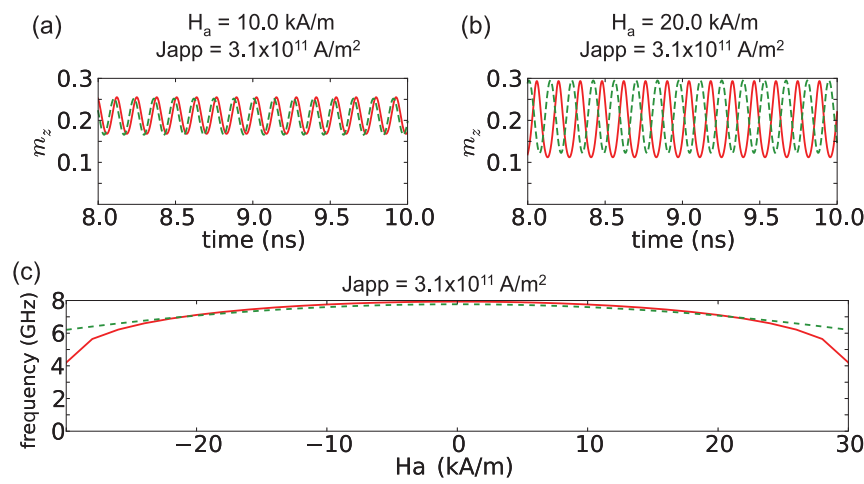

FIG. 4. (Color online) Macrospin simulation (red full line) and analytical expression at first order in $v_{x}$ (green dashed line). (a) and (b) Out-of plane component of the magnetization $m_{z}$ vs time, with applied current density of $J_{\text {app }}=3.1 \times 10^{11} \mathrm{~A} / \mathrm{m}^{2} \quad\left(z_{0}=0.19\right)$ and with applied field (a) $H_{x}=10 \mathrm{kA} / \mathrm{m}\left(v_{x}=0.044\right)$ and (b) $H_{x}=20 \mathrm{kA} / \mathrm{m}$ $\left(v_{x}=0.088\right)$. (c) Frequency of the in-plane component $m_{x}$ vs applied field $H_{x}$.

Figure 4 shows the comparison with macrospin simulations using the same numerical values as previously, except that $J_{\text {app }}=3.1 \times 10^{11} \mathrm{~A} / \mathrm{m}^{2}$ and with an in-plane applied field along $\boldsymbol{u}_{x}$. Figures 4(a) and 4(b) represent the out-of-plane component of the magnetization $m_{z}$ after the transitory regime, for $H_{x}=10 \mathrm{kA} / \mathrm{m}\left(v_{x}=0.044\right)$ and $H_{x}=20 \mathrm{kA} / \mathrm{m}\left(v_{x}=\right.$ 0.088), respectively. In both cases, the analytical expression is in good agreement with the simulations. The frequency versus applied field, in Fig. 4(c), shows also a good agreement between the simulations and the approximation at second order in $v_{x}$ up to $H_{x}=20 \mathrm{kA} / \mathrm{m}$.

Concerning the harmonics of the magnetoresistive signal, the second harmonic has a nonvanishing amplitude, using the formula of the previous section. Here, we consider that the applied current density is large enough so that $\beta \ll 1$ and small enough so that $z_{0}^{2} \ll 1$. For $i=(x, y)$, the two directions of the in-plane applied field are

$$
\begin{aligned}
m_{x}= & r_{0} \cos \tau+\frac{\nu_{i}}{2}\left[\left(z_{0} a_{1}^{i}-r_{0} d_{1}^{i}\right) \cos (2 \tau)\right. \\
& \left.+\left(z_{0} b_{1}^{i}+r_{0} c_{1}^{i}\right) \sin (2 \tau)\right] .
\end{aligned}
$$

Let $\psi$ be a constant phase. The in-plane component $m_{x}$ is approximately given by

$$
m_{x} \approx r_{0} \cos \tau+\frac{\nu_{i}}{2 z_{0}} \cos (2 \tau+\psi) .
$$

\section{Spin torque from the reference layer}

In this section, we focus on the perturbation due to the spin torque of the longitudinal polarizer, initially with $\lambda_{x}=0$. We introduce the variable

$$
\xi=\frac{\alpha}{1+\alpha^{2}} \frac{p_{x}}{p_{z}} .
$$

The associated perturbation functions are given by

$$
\begin{gathered}
f_{\theta}^{\xi}(\theta, \phi)=-\cos \theta \cos \phi-\alpha \sin \phi, \\
f_{\phi}^{\xi}(\theta, \phi)=+\sin \phi-\alpha \cos \theta \cos \phi .
\end{gathered}
$$


In experiments, the reference layer is most often separated from the free layer by a tunnel barrier, whereas the perpendicular polarizer is separated by a metallic barrier. ${ }^{19,20}$ This choice is made in order to maximize the amplitude of the output TMR signal. Consequently, the ratio $r=p_{x} / p_{z}$ is greater than unity. The variable $\xi$ can only be treated as a perturbation because of the $\alpha$ coefficient. Therefore the effect of the longitudinal polarizer on the OPP state is stronger than for other contributions. The solutions are given by

$$
\begin{gathered}
f_{1}=0, \quad f_{2}=\frac{\left(1+\alpha^{2}\right)^{2} z_{0}^{2}}{2\left(1+\alpha^{2} z_{0}^{2}\right)}, \\
\left\{\begin{array}{l}
\theta_{1}\left(\tau^{\prime}\right)=a_{1} \cos \tau^{\prime}+b_{1} \sin \tau^{\prime}, \\
\phi_{1}\left(\tau^{\prime}\right)=c_{1} \cos \tau^{\prime}+d_{1} \sin \tau^{\prime},
\end{array}\right.
\end{gathered}
$$

with the coefficients defined by:

$$
\begin{aligned}
a_{1} & =-\frac{\alpha+\beta z_{0}}{1+\beta^{2}}, \\
b_{1} & =\frac{\alpha \beta-z_{0}}{1+\beta^{2}}, \\
c_{1} & =\frac{\alpha+\beta z_{0}}{\alpha r_{0}\left(1+\beta^{2}\right)}, \\
d_{1} & =-\frac{\alpha \beta+\alpha^{2} z_{0}+z_{0} \beta^{2}\left(1+\alpha^{2}\right)}{\alpha r_{0}\left(1+\beta^{2}\right)} .
\end{aligned}
$$

The comparison with macrospin simulations with $J_{\text {app }}=$ $1 \times 10^{11} \mathrm{~A} / \mathrm{m}^{2}$ is shown in Fig. 5. The analyzer spin polarization $\eta_{\mathrm{LONG}}$ is $\eta_{\mathrm{LONG}}=r \times \eta_{\mathrm{PERP}}=0.2 r$, where $r$ is the ratio $p_{x} / p_{z}$ that varies from 0 to 80 in the simulations. This should give values of $\eta_{\text {LONG }}$ larger than 1, which are unphysical, but they correspond to physical situations by rescaling the applied current density. Hence the absolute value of $\eta_{\mathrm{LONG}}$ is not so important, and only $r$ matters. Figures 5(a) and 5(b) show $m_{z}$ versus time for $r=10(\xi=0.2)$ and $r=20(\xi=0.4)$ respectively. For $r=10$, the analytical expression is offset compared to the simulations, showing that there must be a
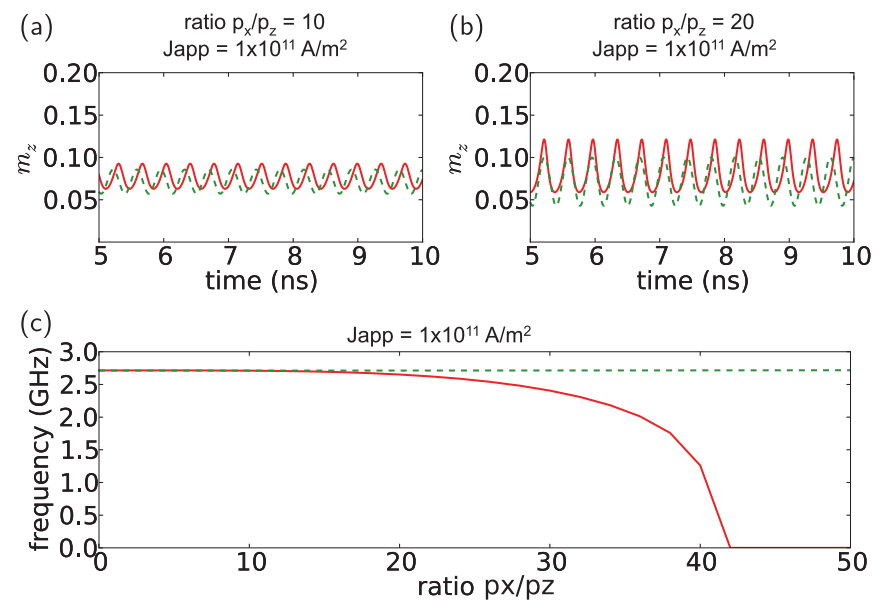

FIG. 5. (Color online) Macrospin simulation (red full line) and analytical expression at first order in $\xi$ (green dashed line). (a) and (b) Out-of plane component of the magnetization $m_{z}$ vs time, with applied current density of $J_{\text {app }}=1 \times 10^{11} \mathrm{~A} / \mathrm{m}^{2}\left(z_{0}=0.06\right)$ and with a ratio $r=p_{x} / p_{z}$ (a) $r=10(\xi=0.2)$ and (b) $r=20(\xi=0.4)$. (c) Frequency of the in-plane component $m_{x}$ vs ratio $p_{x} / p_{z}$. constant term arising from the second-order development that is not taken into account here. For $r=20$, the simulated $m_{z}$ is not sinusoidal, giving rise to a frequency mismatch with the first-order analytical expression. The frequency change versus ratio $r$ shown in Fig. 5(c) shows that it is at least a third-order perturbation, as second-order frequency change cannot explain the frequency drop for large $r$.

Because the perturbation in first order has a frequency that is the fundamental frequency, like for the applied field, the second harmonic amplitude is nonzero:

$$
m_{x} \approx r_{0} \cos \tau+2 \xi \cos \left(2 \tau+\psi^{\prime}\right) .
$$

Here, $\beta \ll 1$ and $z_{0}^{2} \ll 1$ were assumed, and $\psi^{\prime}$ is a constant phase.

\section{Interplay of two contributions}

The power series expansion was only carried up to second order, because of the difficulty of developing further the analytical solutions and also because second order is sufficient regarding the negligible size of other terms. So far, only one contribution was considered at a time, but if we consider two simultaneous contributions, the perturbed expressions are changed. In first order, the influence of the different contributions can be added directly to determine the analytical expression for the angle $\theta$ and $\phi$, there is no interaction between the contributions. However, at second order, the different contributions do not simply add together; there is also an additional term of interaction. Sometimes the interaction term vanishes, for instance, with the uniaxial anisotropy combined with the longitudinal spin torque or the applied field. However, if the longitudinal spin torque $p_{x}$ and the applied field $H_{x}$ (similar for $H_{y}$ ) are taken into account, an interaction term is added to the perturbed frequency.

We perform an expansion by setting $\zeta=\frac{H_{x}}{p_{x}}$ and expanding with respect to $\xi$. The frequency correction is given by the term coming from $H_{x}$ alone multiplied by $v_{x}^{2}$, the term coming from $p_{x}$ alone multiplied by $\xi^{2}$, plus the interaction term, multiplied by $\xi v_{x}$ :

$$
f_{2}=-\frac{\left(1+\alpha^{2}\right)^{3} z_{0}^{2}}{\alpha\left(1+\alpha^{2} z_{0}^{2}\right)\left(\alpha^{2}+z_{0}^{2}\right)} .
$$

We can also treat the case of an angle-dependant spin torque amplitude with uniaxial anisotropy. First, we replace $p_{z}$ by $p_{z} /\left(1+\lambda_{z} \cos \theta\right)$ and treat $\lambda_{z}$ as a perturbative parameter. Without any other perturbation, the variation of the frequency was derived exactly in the high-symmetry case, $f=f_{0} /(1+$ $\left.\lambda_{z} z_{+}\right)$, and its Taylor expansion versus $\lambda_{z}$ corresponds to the terms derived by the technique used here. However, there is an interaction with the uniaxial anisotropy term at the second order. On top of the uniaxial anisotropy and the spin torque asymmetry, $2 \lambda_{z}^{2} z_{0}^{2}$, terms, there is an interaction term multiplied by $\lambda_{z} \epsilon$ :

$$
f_{2}=z_{0}^{2}\left(1+\alpha^{2}\right) .
$$

Finally, we study the case of an angle-dependent spin torque along the $x$ axis. There is an interaction between the angleindependent term $p_{x}$ and the asymmetry parameter $\lambda_{x}$. For this we use the Taylor expansion versus $\lambda_{x}$ of the full expression $\frac{p_{x} \sin \theta \cos \phi}{1+\lambda_{x} \sin \theta \cos \phi}$. We compute the perturbation approximation by 
expanding with the parameter $\xi$, and by $\lambda_{x}$ if necessary. The frequency change is given by the term on $\xi$ only, plus a firstorder and a second-order terms depending on $\lambda_{x}$ also:

$$
\begin{aligned}
f \approx & f_{0}\left[1+\xi \lambda_{x} \frac{z_{0}}{2 \alpha}\left(1+\alpha^{2}\right)+\xi^{2} \lambda_{x}^{2} \frac{z_{0}^{2}}{4 \alpha^{2}}\left(1+\alpha^{2}\right)^{2}\right. \\
& \left.+\xi^{2} \frac{\left(1+\alpha^{2}\right)^{2} z_{0}^{2}}{2\left(1+\alpha^{2} z_{0}^{2}\right)}\right] .
\end{aligned}
$$

\section{CRITICAL CURRENTS}

\section{A. Stability criterion}

The expressions of the OPP trajectory with perturbation up to first order define a stability criterion for these trajectories. Regarding the Poincaré's map multiplier for the trajectories up to first order, $\left|\mu_{1}\right|<1$ for all the perturbations treated in this paper, regardless of the amplitude of the perturbation. It means that the corrected trajectories are stable to sufficiently small thermal fluctuations. However, the multiplier does not give any information on the maximum amplitude of the perturbations up to which the out-of-plane precession is maintained.

For this we use the Melnikov function. ${ }^{8,21}$ This function can be extracted from the equation of motion (4). By operating the vectorial product of this equation by $\mathrm{d} \boldsymbol{m}$, then taking the scalar product with $\boldsymbol{m}$, we get

$$
\left(\boldsymbol{m} \times \nabla_{\boldsymbol{m}} \mathcal{G}+\nabla_{\boldsymbol{m}} \mathcal{H}\right) \cdot \mathrm{d} \boldsymbol{m}=0 .
$$

If the trajectory of the system is periodic, the closed integral of the Hamiltonian part of the previous expression vanishes, namely,

$$
\oint_{\Gamma} \nabla_{\boldsymbol{m}} \mathcal{H} \cdot \mathrm{d} \boldsymbol{m}=0
$$

Only the second part of the expression remains, $\oint\left(\boldsymbol{m} \times \nabla_{\boldsymbol{m}} \mathcal{G}\right)$. $\mathrm{d} \boldsymbol{m}=0$. Using the expression of $\mathrm{d} \boldsymbol{m}$ from the equation of motion, we obtain

$$
\mathcal{M}(\Gamma)=\oint_{\Gamma}\left[\nabla_{\boldsymbol{m}} \mathcal{H} \cdot \nabla_{\boldsymbol{m}} \mathcal{G}-\left(\boldsymbol{m} \cdot \nabla_{\boldsymbol{m}} \mathcal{H}\right)\left(\boldsymbol{m} \cdot \nabla_{\boldsymbol{m}} \mathcal{G}\right)\right] d t=0
$$

The above integral is called the Melnikov function. If the path $\Gamma$ follows a periodic orbit solution of the equation of motion, then the Melnikov function vanishes. In the framework of weakly perturbed time periodic Hamiltonian systems, the Melnikov function is approximated by evaluating the closed loop integral over the trajectory $\Gamma_{0}$ of the unperturbed system $(\mathcal{G}=0)$ with constant energy $w$. The condition that the Melnikov function must vanish for a periodic orbit gives a constraint on the parameters of the system, namely the current density $J$.

The issue of calculating the critical parameters for which the periodic orbit is maintained has already been addressed by Ebels et al. ${ }^{8}$ in the case of a free layer with a perpendicular polarizer and an applied field along the easy axis ( $x$ axis). They use a similar technique of zeroing the Melnikov function to obtain the critical current $J_{c 4}$ below which no periodic orbit is possible. It is important to be precise that the present paper focuses on determining the critical current $J_{c 4}$ and not $J_{c 1}$, of which the origin and expression come from stability conditions of the in-plane equilibrium state. Ebels et al. ${ }^{8}$ extracted numerical data for the critical current $J_{c 4}$ with respect to the applied field, the anisotropy field $H_{k}$ (named $H_{u}$ in Ref. 8), the saturation magnetization $M_{S}$, and the damping constant $\alpha$. Unfortunately, no analytical expression was found.

Critical currents for stability of the periodic out-of-plane precession can be derived using the expressions computed in the previous section. Using the expressions of the angles $\theta$ and $\phi$ at first order in the perturbation parameter, we can check that the in-plane angle variation $\dot{\phi}$ never vanishes. Otherwise, the precessional motion would be stopped. Using this criterion, critical spin torque amplitudes were computed, with only one perturbation at a time. The critical current $J_{c}$ is proportional to the critical spin torque amplitudes $p_{z_{c}}$. The critical spin torque amplitudes are given beneath for the different perturbation cases, respectively, uniaxial anisotropy, in-plane applied field (there is no distinction between $H_{x}$ and $H_{y}$ direction, so we use $H_{a}$ pointing in any arbitrary in-plane direction), and spin torque from the longitudinal polarizer. Here, we considered the case where $\alpha^{2} \ll\left(H_{a} / M_{s}, H_{k} / M_{s}\right)$, which is more common in experiments, so that $\beta \ll 1$.

For the uniaxial anisotropy and the applied field, the out-of-plane precessional state is allowed as long as $p_{z}>p_{z_{c}}$. For these two perturbations, the stability criterion defines a minimal spin torque amplitude for the existence of the precessional state:

$$
\begin{gathered}
\text { anisotropy: } \quad p_{z_{c}}=\frac{\alpha}{2} \sqrt{H_{k} M_{S}}, \\
\text { in-plane applied field : } \quad p_{z_{c}}=\alpha \sqrt{H_{a} M_{S}} .
\end{gathered}
$$

Figures 6 and 7 present the average in-plane component $m_{x}$ of the free-layer magnetization with an applied current pulse of $10 \mathrm{~ns}$ from macrospin simulations. The average is taken between 8 and $10 \mathrm{~ns}$ to avoid the transient regime. The magnetization is initially almost completely out-of-plane $\left(m_{z} \approx 1\right)$ to favor the OPP steady state that appears when the magnetization relaxes towards the plane. The average magnetization is represented versus the applied current density, the anisotropy field in Fig. 6, and the in-plane applied field along $\boldsymbol{u}_{x}$ in Fig. 7. The blue and red colors correspond to an average magnetization in equilibrium in the plane, $\left\langle m_{x}\right\rangle= \pm 1$, respectively. The light green color corresponds

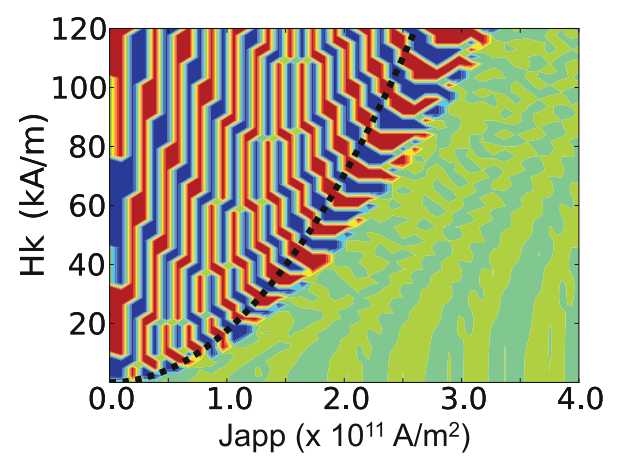

FIG. 6. (Color online) From macrospin simulations, the average in-plane component $m_{x}$ of the magnetization in permanent regime vs applied current density and anisotropy field. Dark blue and red colors correspond to the equilibrium states $\left\langle m_{x}\right\rangle= \pm 1$, respectively. Light green color corresponds to $\left\langle m_{x}\right\rangle=0$, the out-of-plane precessional steady state. The analytical critical current is represented by the black dashed line. 


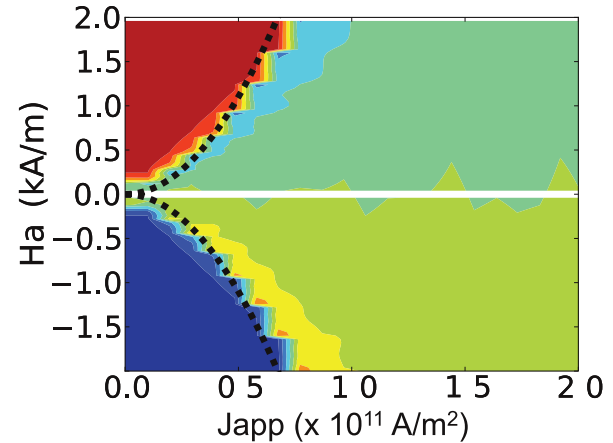

FIG. 7. (Color online) Same as Fig. 6 with the applied field along $\boldsymbol{u}_{x}$.

to $\left\langle m_{x}\right\rangle=0$, representative of the OPP steady state. We can extract the critical current $J_{c 4}$ from the boundary between light green and dark blue/red regions. The analytical critical line from expression (27) is represented in black dashed line. For the highest value of anisotropy and applied fields used in the simulations, the analytical critical current differs from the simulations value by $20 \%$ and $10 \%$, respectively. Notice that for the anisotropy figure, the equilibrium region is not uniformly colored and presents blue and red stripes because depending on where the OPP is stopped, the magnetization relaxes to $m_{x}=-1$ or 1 . The final states actually depend on the transient regime, they are of little interest here. The critical currents obtained by finding numerically the root of the Melnikov function, as in Ebels et al., ${ }^{8}$ are also in agreement with the analytical expressions. In fact, they match with an error of less than $10 \%$ as seen on Fig. 8, which represents the critical spin torque amplitude find by numerical methods, normalized by the analytical expression, versus applied field.

For the analyzer spin torque, the same criterion used for anisotropy and applied field defines a maximal spin torque amplitude for which the periodic orbit exists. The critical current density depends on the ratio $r=p_{x} / p_{z}$ of the two spin torque contributions:

$$
\begin{array}{ll}
p_{z_{c}}=\alpha M_{S}\left(1-\alpha^{2} r^{2}\right) & \text { for } \quad 2 \alpha r<1, \\
p_{z_{c}}=\frac{\alpha^{2} M_{S}}{\sqrt{4 \alpha^{2} r^{2}-1}} & \text { for } \quad 2 \alpha r>1 .
\end{array}
$$

The phase diagram of Fig. 9 represents the average of the in-plane magnetization $m_{x}$ from macrospin simulations with different applied current density and ratio $r=p x / p z$. The analytical expression for the critical current density is drawn in black dashed line. The analytical critical line corresponds

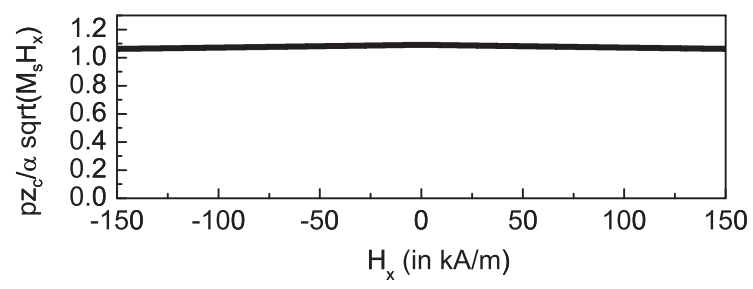

FIG. 8. Critical spin torque amplitude computed with the Melnikov function vs applied field $H_{x}$ along $\boldsymbol{u}_{x}$. The critical spin torque amplitude is normalized by the analytical expression.

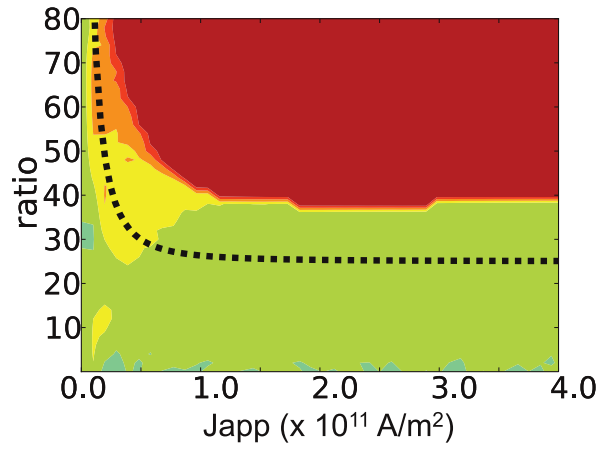

FIG. 9. (Color online) Same as Fig. 6 with the ratio $r=p_{x} / p_{z}$.

qualitatively to the region boundary of the diagram. However, for a given current density, the corresponding critical ratio is $35 \%$ smaller with the analytical expression than found by macrospin simulations. On the critical line, the ratio is around 40 , giving a perturbation parameter $\xi=0.8$. In this range, we should consider higher orders to obtain a better accuracy on the critical current.

\section{DISCUSSION}

The perturbative approach developed here shows good agreement with macrospin simulations even at low order. For the anisotropy and the applied fields, we were able to calculate the frequency change due to these perturbations, except close to the range where the frequency vanishes. For these perturbations, we were also able to give a good approximation of the critical current $J_{c 4}$ at which the OPP state disappears. In fact, the perturbation parameter, for instance $\epsilon$, is more or less inversely proportional to the frequency of the OPP, so it is clear that the perturbative expansion is less precise when the frequency vanishes, or close to the critical current, which is determined by this same criterion. Another limit is when the applied current density tends towards zero, that corresponds also to a vanishing frequency. However, this limit is not very relevant physically as the dynamical system studied here presents another critical current density, usually called $J_{c 1}$, below which the magnetization remains in static equilibrium in-plane. So the dynamical system presents a bifurcation between an equilibrium and a limit cycle not associated with this equilibrium, and the frequency is nonvanishing close to the critical current $J_{c 1}$.

This study has been carried out for an in-plane magnetized MTJ with additional perpendicular polarizer. The same perturbation expansion could also be done with a MTJ with in-plane precession (IPP) steady state. A good starting point to treat this case would be to consider the LandauLifshitz-Gilbert equation in complex numbers as described by the Kim, Tiberkevich, and Slavin (KTS) theory; ${ }^{10-12}$ if we write the complex differential equation as a couple of differential equations for the amplitude and the phase, the amplitude would play the role of $\theta$ here, and the phase the role of $\phi$. By rescaling the time with the eigenfrequency $\omega_{0}$ corresponding to the FMR frequency, we expect to carry out the Lindstedt's series expansion of the trajectory with constant energy, considering $\alpha$ as a small parameter. It should give a good approximation of the nonlinear parameters modifying the 
frequency and the linewidth, and also the relative amplitude of the second harmonics, which are not treated by the present theory. However, this is beyond the scope of this paper.

\section{ACKNOWLEDGMENT}

This work was supported by the European commission through the ERC Adv Grant HYMAGINE No. 246942.

\section{APPENDIX: LINDSTEDT'S SERIES EXPANSION}

The Lindstedt series expansion is a method used to obtain an approximation of the solution of a dynamical system $(S)$ with a small perturbation, by using the solution of an unperturbed system $\left(S_{0}\right)$. Let $(S)$ and $\left(S_{0}\right)$ be the following systems, and $\boldsymbol{X}_{0}$ be the solution of $\left(S_{0}\right)$ :

$$
\begin{aligned}
(S): & \dot{\boldsymbol{X}} & =\boldsymbol{F}(\boldsymbol{X})+\epsilon \boldsymbol{G}(\boldsymbol{X}), \\
\left(S_{0}\right): & \dot{\boldsymbol{X}}_{\mathbf{0}} & =\boldsymbol{F}\left(\boldsymbol{X}_{0}\right) .
\end{aligned}
$$

In order to find the solution of $(S)$, we suppose that the solution $\boldsymbol{X}$ of $(S)$ can be written as a power series of the small parameter $\epsilon$ :

$$
\boldsymbol{X}=\boldsymbol{X}_{0}+\epsilon \boldsymbol{X}_{1}+\epsilon^{2} \boldsymbol{X}_{2}+\ldots .
$$

We can then replace $\boldsymbol{X}$ by the following power series in the system $(S)$ :

$$
\begin{aligned}
\dot{\boldsymbol{X}}_{0}+\epsilon \dot{\boldsymbol{X}}_{1}+\epsilon^{2} \dot{\boldsymbol{X}}_{2}+\ldots= & \boldsymbol{F}\left(\boldsymbol{X}_{0}+\epsilon \boldsymbol{X}_{1}+\epsilon^{2} \boldsymbol{X}_{2}+\ldots\right) \\
& +\epsilon \boldsymbol{G}\left(\boldsymbol{X}_{0}+\epsilon \boldsymbol{X}_{1}+\epsilon^{2} \boldsymbol{X}_{2}+\ldots\right) .
\end{aligned}
$$

Then the perturbed dynamical system $(S)$ becomes $\left(S_{1}\right)$ at the first order in $\epsilon$ :

$$
\left(S_{1}\right): \quad \dot{\boldsymbol{X}}_{0}+\epsilon \dot{\boldsymbol{X}}_{1}=\boldsymbol{F}\left(\boldsymbol{X}_{0}\right)+\epsilon \mathrm{d} \boldsymbol{F}\left(\boldsymbol{X}_{0}\right)\left[\boldsymbol{X}_{1}\right]+\epsilon \boldsymbol{G}\left(\boldsymbol{X}_{0}\right) .
$$

Here, $\mathrm{d} \boldsymbol{F}\left(\boldsymbol{X}_{0}\right)$ is the differential of the vector field $\boldsymbol{F}$ evaluated at $\boldsymbol{X}_{0}$. By identification, we obtain two differential equations, where the first one is the unperturbed system $\left(S_{0}\right)$ :

$$
\begin{aligned}
\dot{\boldsymbol{X}}_{0} & =\boldsymbol{F}\left(\boldsymbol{X}_{0}\right), \\
\dot{\boldsymbol{X}}_{1} & =\mathrm{d} \boldsymbol{F}\left(\boldsymbol{X}_{0}\right)\left[\boldsymbol{X}_{1}\right]+\boldsymbol{G}\left(\boldsymbol{X}_{0}\right) .
\end{aligned}
$$

This method theoretically allows to calculate the solution up to any order. However, if the solution $\dot{\boldsymbol{X}}_{k}$ at order $k$ is periodic, the resolution of the dynamical system at order $k+1$ may give rise to nonperiodic secular terms, often $t \cos \left(\omega_{0} t\right)$ terms, or, for the equation addressed in this paper, $t$ terms. In order to get rid of the secular terms, we also suppose that the angular frequency can be written as a power series of $\epsilon: \omega=\omega_{0}+\epsilon \omega_{1}+\epsilon^{2} \omega_{2}+\ldots$. By rescaling time with the change of variables $\tau=\omega t$, we rewrite system $(S)$ as

$$
\begin{gathered}
\left(\omega_{0}+\epsilon \omega_{1}+\epsilon^{2} \omega_{2}+\ldots\right)\left(\dot{\boldsymbol{X}}_{0}+\epsilon \dot{\boldsymbol{X}}_{1}+\epsilon^{2} \dot{\boldsymbol{X}}_{2}+\ldots\right) \\
=\boldsymbol{F}\left(\boldsymbol{X}_{0}+\epsilon \boldsymbol{X}_{1}+\epsilon^{2} \boldsymbol{X}_{2}+\ldots\right) \\
\quad+\epsilon \boldsymbol{G}\left(\boldsymbol{X}_{0}+\epsilon \boldsymbol{X}_{1}+\epsilon^{2} \boldsymbol{X}_{2}+\ldots\right) .
\end{gathered}
$$

Henceforth, $\dot{\boldsymbol{X}}$ denotes a derivation with respect to $\tau$. The first-order system becomes

$$
\dot{\boldsymbol{X}}_{1}+\omega_{1} \dot{\boldsymbol{X}}_{0}=\mathrm{d} \boldsymbol{F}\left(\boldsymbol{X}_{0}\right)\left[\boldsymbol{X}_{1}\right]+\boldsymbol{G}\left(\boldsymbol{X}_{0}\right) .
$$

The value of $\omega_{1}$, and higher-order terms $\omega_{k}$, is set so that secular terms are canceled. In the majority of the cases treated in this paper, it appears that $\omega_{1}=0$ because no secular terms appear at order 1 . However, at order 2 , in order to cancel the secular terms, $\omega_{2}$ needs to be nonzero.

However, this way of solving the perturbed system does not account for an additional temporal dephasing between the angles $\theta$ and $\phi$. According to the exact solutions of Eq. (8), the second term of the right-hand side from the expression of $\phi$ is a dephasing term that accounts for the initial condition $z_{i}$. In this particular case, the angle $\theta$ is constant in the permanent regime so this dephasing has no effect. However, solving a perturbed system gives rise to oscillating terms on the angle $\theta$, for which the exact dephasing with the angle $\phi$ is important, specially for second-order terms. In fact, the second-order terms partly account for an interaction between $\theta$ and $\phi$ harmonics. In order to solve this issue, we first calculate the equation of the angle $\theta$ with respect to $\phi$, then the expression of $\theta(\tau)$ and $\phi(\tau)$. By dividing the first equation of Eq. (16) by the second equation, we obtain the differential equation governing $\theta(\phi)$ :

$$
\frac{\mathrm{d} \theta}{\mathrm{d} \phi}=\sin \theta \frac{g_{\theta}(\theta)+\epsilon f_{\theta}^{\epsilon}(\theta, \phi)}{h_{\phi}(\theta)+\epsilon f_{\phi}^{\epsilon}(\theta, \phi)} .
$$

Both sides of this equation can be Taylor expanded with respect to $\epsilon$ by using the expressions for $\theta$ and $\phi$ from Eq. (17). Hence the expression of $\theta$ with respect to $\phi$ is computed up to any given order $k$. The assumption that $\epsilon \ll 1$, or that the perturbation is small compared to the nonperturbated frequency, is critical here.

Thereafter, the expression of $\theta(\phi)$ at order $k$ is injected in the differential equations (16) of $\theta$ and $\phi$ versus time $\tau$ to obtain the precession harmonics and the frequency changes, using the Lindstedt's series.

The results of Sec. III were obtained with the computer algebra system MAXIMA, ${ }^{22}$ by differentiating Eq. (A1) with respect to $\epsilon$ to get the equations at the different orders in $\epsilon$. At order 2, the solutions already hold many terms that are not shown here because we were only concerned about the frequency change. The computation of higher-order terms is rather complicated.
${ }^{1}$ O. Redon, B. Dieny, and B. Rodmacq, Patent No. US6532164B2 (2001).

${ }^{2}$ J. A. Katine and E. E. Fullerton, J. Mag. Mag. Mater. 320, 1217 (2008).

${ }^{3}$ Z. Li and S. Zhang, Phys. Rev. B 68, 024404 (2003).
${ }^{4}$ D. C. Ralph and M. D. Stiles, J. Mag. Mag. Mater. 320, 1190 (2008).

${ }^{5}$ S. I. Kiselev, J. C. Sankey, I. N. Krivorotov, N. C. Emley, R. J. Schoelkopf, R. A. Buhrman, and D. C. Ralph, Nature (London) 425, 380 (2003).

${ }^{6}$ D. Houssameddine, U. Ebels, B. Delat, B. Rodmacq, I. Firastrau, F. Ponthenier, M. Brunet, C. Thirion, J.-P. Michel, 
L. Prejbeanu-Buda, M.-C. Cyrille, O. Redon, and B. Dieny, Nat. Mater. 6, 441 (2007).

${ }^{7}$ A. Vaysset, C. Papusoi, L. D. Buda-Prejbeanu, S. Bandiera, M. Marins de Castro, Y. Dahmane, J.-C. Toussaint, U. Ebels, S. Auffret, R. Sousa, L. Vila, and B. Dieny, Appl. Phys. Lett. 98, 242511 (2011).

${ }^{8}$ U. Ebels, D. Houssameddine, I. Firastrau, D. Gusakova, C. Thirion, B. Dieny, and L. D. Buda-Prejbeanu, Phys. Rev. B 78, 024436 (2008).

${ }^{9}$ K. J. Lee, O. Redon, and B. Dieny, Appl. Phys. Lett. 86, 022505 (2005).

${ }^{10}$ A. N. Slavin and V. Tiberkevich, Magnetics, IEEE Transactions On 44, 1916 (2008).

${ }^{11}$ A. N. Slavin and V. Tiberkevich, Magnetics, IEEE Transactions On 45, 1875 (2009).

${ }^{12}$ J. V. Kim, V. Tiberkevich, and A. N. Slavin, Phys. Rev. Lett. 100, 017207 (2008).
${ }^{13}$ Y. B. Bazaliy, Phys. Rev. B 85, 014431 (2012).

${ }^{14}$ J. C. Slonczewski, J. Mag. Mag. Mater. 247, 324 (2002).

${ }^{15}$ J. C. Slonczewski, Phys. Rev. B 71, 024411 (2005).

${ }^{16} \mathrm{~T}$. Silva and M. Keller, Magnetics, IEEE Transactions On 46, 3555 (2010).

${ }^{17}$ G. Bertotti, I. D. Mayergoyz, and C. Serpico, Physica B 306, 47 (2001).

${ }^{18}$ J. Murdoch, Perturbations: Theory and Methods (Wiley, New York, 1991).

${ }^{19}$ H. Liu, D. Bedau, D. Backes, J. A. Katine, J. Langer, and A. D. Kent, Appl. Phys. Lett. 97, 242510 (2010).

${ }^{20}$ M. Marins de Castro, R. C. Sousa, S. Bandiera, C. Ducruet, A. Chavent, S. Auffret, C. Papusoi, I. L. Prejbeanu, C. Portemont, L. Vila, U. Ebels, B. Rodmacq, and B. Dieny, J. Appl. Phys. 111, 07C912 (2012).

${ }^{21}$ Y. B. Bazaliy, Phys. Rev. B 76, 140402 (2007).

${ }^{22} \mathrm{http}: / /$ maxima.sourceforge.net/. 